\title{
Behavioral momentum and relapse of extinguished operant responding
}

\author{
Christopher A. Podlesnik and Timothy A. Shahan \\ Utah State University, Logan, Utah
}

\begin{abstract}
Previous experiments on behavioral momentum have shown that relative resistance to extinction of operant behavior in the presence of a stimulus depends on the rate of reinforcement associated with that stimulus, even if some of those reinforcers occur independently of the behavior. We present three experiments examining whether the rate of reinforcement in the presence of a stimulus similarly modulates the relative relapse of operant behavior produced by reinstatement, resurgence, and renewal paradigms. During baseline conditions, pigeons responded for food reinforcement on variable-interval 120 -sec schedules in alternating periods of exposure to two stimuli arranged by a multiple schedule. Additional response-independent food presentations were also delivered in the presence of one of the multiple-schedule stimuli. Consistent with previous research, baseline response rates were lower in the presence of the stimulus with the added response-independent reinforcement, and relative resistance to extinction was greater in the presence of that stimulus. In addition, following extinction, the relative relapse of responding produced by reinstatement, resurgence, and renewal paradigms was greater in the presence of the stimulus associated with the higher rate of reinforcement. We suggest that a model of extinction from behavioral momentum theory may be useful for understanding these results.
\end{abstract}

Behavioral momentum theory provides a theoretical framework for understanding how differential reinforcement conditions affect the resistance to change (i.e., strength or persistence) of discriminated operant behavior (Nevin \& Grace, 2000). Resistance to change is usually examined with multiple schedules of reinforcement in which different rates or magnitudes of reinforcement are arranged in the presence of distinctive stimuli that alternate throughout the experimental session. Once a baseline of performance is established, responding is disrupted with extinction or some other operation. The resulting decrease in response rates for the two stimuli, relative to predisruption baseline response rates (i.e., proportion of baseline response rates), provides a measure of resistance to change. Relatively smaller decreases from baseline reflect behavior that is more resistant to change. A general finding is that responding is more resistant to change in the presence of stimuli associated with higher rates or greater magnitudes of reinforcement (see Nevin, 1992).

Behavioral momentum theory suggests that response rates and resistance to change are separable aspects of operant behavior. The contingent response-reinforcer relation governs response rate (e.g., Herrnstein, 1970), but the Pavlovian stimulus-reinforcer relation between a discriminative stimulus and reinforcers obtained during that stimulus governs resistance to change. Formally, relative resistance to change in the presence of two stimuli is a power function of the relative rates of reinforcement arranged in the presence of those stimuli, so that

$$
m_{1} / m_{2}=\left(r_{1} / r_{2}\right)^{b},
$$

where $m_{1}$ and $m_{2}$ are the resistance to change of responding to Stimuli 1 and 2 and $r_{1}$ and $r_{2}$ refer to the rates of reinforcement delivered in the presence of those stimuli (Nevin, 1992). The parameter $b$ reflects sensitivity of ratios of resistance to change in the presence of the two stimuli to variations in the ratio of reinforcement rates for the two stimuli. The reinforcers in the right-hand side of Equation 1 need not be contingent upon the behavior in question in order to increase relative resistance to change.

Nevin, Tota, Torquato, and Shull (1990) provided support for the separable roles of the response-reinforcer and stimulus-reinforcer relations in the control of response rates and resistance to change, respectively. In two experiments, Nevin et al. examined the effects of adding either response-independent reinforcement or reinforcement contingent upon a concurrently available behavior on response rates and resistance to change. By improving the stimulus-reinforcer relation, the added reinforcers were expected to increase resistance to change, regardless of their impact on response rates. Consistent with this expectation, Nevin et al. found that the added reinforcers decreased response rates but increased resistance to change. This result has been replicated in similar experiments using a variety of responses, reinforcers, and species ranging 
from fish to humans (Ahearn, Clark, Gardenier, Chung, \& Dube, 2003; Cohen, 1996; Grimes \& Shull, 2001; Harper, 1999; Igaki \& Sakagami, 2004; Mace et al., 1990; Shahan \& Burke, 2004).

Although previous research on behavioral momentum has documented the impact of the stimulus-reinforcer relation on resistance to disruption of operant behavior, an additional feature of persistent behavior is its tendency to relapse after extinction. Given the obvious clinical relevance of relapse, a considerable amount of recent research has been directed at phenomena characterized by the reappearance of behavior after extinction.

The most widely examined relapse-related phenomenon with operant behavior is reinstatement. Reinstatement of extinguished operant behavior occurs when the presentation of the reinforcer maintaining responding prior to extinction is response independent and the extinguished responding reappears (e.g., Reid, 1958). The reinstatement paradigm has been used extensively to study the reappearance of extinguished drug seeking in animals and is widely accepted as an animal model of relapse in substance abuse research (for reviews, see See, 2002; Shaham, Shalev, Lu, de Wit, \& Stewart, 2003).

Another relapse-related phenomenon resulting in the reappearance of extinguished operant behavior is resurgence. Resurgence is characterized by the reappearance of an extinguished operant response when an alternative behavior introduced during extinction is subsequently placed on extinction (e.g., Leitenberg, Rawson, \& Bath, 1970; Leitenberg, Rawson, \& Mulick, 1975; Podlesnik, Jimenez-Gomez, \& Shahan, 2006; see also Epstein, 1996). For example, leverpressing by a rat maintained by food might be placed on extinction at the same time that an alternative response (e.g., chain pulling) is introduced and now produces the food. When chain pulling is also then placed on extinction, the original response of leverpressing reappears.

Finally, renewal refers to a reappearance of extinguished responding that occurs with a change in the context in which extinction took place. Although more frequently examined using Pavlovian-conditioning preparations (see Bouton, 2004, for a review), renewal has also been demonstrated with operant behavior (Nakajima, Tanaka, Urushihara, \& Imada, 2000; Welker \& McAuley, 1978). For example, Nakajima et al. trained rats to press a lever for food on a variable interval (VI) 30 -sec schedule in a chamber characterized by distinct textual, visual, and auditory contextual stimuli (i.e., Context A). Next, leverpressing was extinguished in the presence of different contextual stimuli (i.e., Context B). Finally, leverpressing increased when Context A was reintroduced, even though extinction remained in effect.

The purpose of the present experiments was to examine whether relapse of extinguished operant behavior produced by reinstatement, resurgence, and renewal procedures depends on the baseline stimulus-reinforcer relation in a manner similar to extinction within a behavioral momentum framework. In each experiment, a baseline of operant responding of pigeons in the presence of two stimuli was established within sessions, using a multiple schedule of reinforcement. Responding in the presence of both stimuli produced response-dependent reinforcers at the same frequency. Additional response-independent reinforcers were included during one stimulus in order to improve the Pavlovian stimulus-reinforcer relation defined by that stimulus. In Experiments 1, 2, and 3, relapse following extinction was examined, using reinstatement, resurgence, and renewal paradigms, respectively. The primary question was whether the improvement in the stimulus-reinforcer relation associated with the inclusion of added response-independent reinforcers in the presence of one stimulus would increase both relative resistance to extinction and relative relapse of responding in the presence of that stimulus.

\section{EXPERIMENT 1}

This experiment examined the impact of additional response-independent reinforcer presentations on relative resistance to extinction and relapse produced by a reinstatement preparation. A baseline condition arranged equal VI schedules for an operant response in the presence of two stimuli within a multiple schedule. In the presence of one stimulus, added response-independent reinforcers were also delivered. Following extinction in the presence of both stimuli, reinstatement was assessed by presenting response-independent or response-dependent food presentations at the beginning of the session in each component.

\section{Method}

Subjects. The subjects were 10 unsexed homing pigeons maintained at approximately $80 \%$ of their free-feeding weights $( \pm 15 \mathrm{~g})$ by postsession supplemental feeding of pelleted pigeon food as necessary. All the pigeons had experience with multiple schedules of reinforcement similar to those used in the present experiment and had participated in one or more of the other experiments reported below. The Appendix lists the pigeons serving in each experiment and the order of exposure to the experiments. The pigeons were housed in individual cages in a temperature-controlled room with a $12: 12-\mathrm{h}$ light:dark cycle with lights on at 7:00 a.m. All the pigeons had free access to water in their home cages.

Apparatus. The experiment was conducted in four BRS/LVE pigeon chambers constructed from painted metal with aluminum front panels. Each chamber measured $35 \mathrm{~cm}$ long, $35.8 \mathrm{~cm}$ high, and $30.7 \mathrm{~cm}$ wide. Three translucent plastic response keys were $2.6 \mathrm{~cm}$ in diameter and were located on the front panel $24 \mathrm{~cm}$ above the floor and required a force of approximately $0.1 \mathrm{~N}$ to operate. Only the center key was used. Turning off the houselight for $0.01 \mathrm{sec}$ provided response feedback during each response to a lighted key. This resulted in a brief flicker of the houselight. Reinforcement presentations consisted of 2 -sec access to pelleted pigeon food from a hopper located on the midline of the work panel located with its center $10 \mathrm{~cm}$ from the floor. When raised, the hopper was accessible through a 5 -cm-wide $\times 5.5-\mathrm{cm}$-tall aperture illuminated by a $28-\mathrm{V}$ DC bulb. All other illumination was extinguished while the hopper was operated, and hopper time was excluded from all calculations. A shielded 28-V DC bulb mounted $4.4 \mathrm{~cm}$ above the center key provided general illumination. White noise and a ventilation fan masked extraneous sounds. Control of experimental events and data recording were conducted by a microcomputer using Med Associates interfacing and software.

Procedure. During the baseline condition, a two-component multiple schedule signaled by different colored keylights arranged equal rates of response-dependent food reinforcement for pecks 
to the center key on separate VI 120-sec schedules. Responseindependent food presentations were added to one component on a variable time (VT) 20 -sec schedule. Hereafter, the component with added food will be referred to as the rich component, and the component without added food will be referred to as the lean component. The stimuli used for the two components in this and the subsequent experiments are presented in the Appendix. All schedules of reinforcement comprised eight intervals (Fleshler \& Hoffman, 1962) selected without replacement until all intervals were used. Each 60-sec component was presented 12 times per session and had an equal probability of beginning each session (for exceptions, see below), after which the components strictly alternated. Sessions began with and were separated by 30 -sec intercomponent intervals (ICIs), during which the keylights were dark but the houselight remained on. All the pigeons received 30 sessions of baseline training before beginning the extinction condition. In the extinction condition, all reinforcers in both components were withheld. Thus, both response-dependent reinforcement on the center key in both components and response-independent food presentations in the rich component were discontinued. The reinstatement condition began once response rates on the center key decreased below $10 \%$ of baseline for 2 consecutive sessions in both components.

In the reinstatement condition, food reinforcers were presented response independently or response dependently in separate blocks of four sessions. When response-independent food was assessed, two food presentations occurred, 2 and $8 \mathrm{sec}$ into the first presentation of each component in a session. Reinstatement by responsedependent food reinforcers was also assessed, to serve as a control condition, because response-independent food presentations might generate stimulus conditions more similar to the baseline condition in the rich component. When response-dependent reinforcement was assessed, the first two responses during the first presentation of each component resulted in reinforcement. Extinction was in effect throughout the remainder of the components. The order in which response-independent and response-dependent blocks of reinstatement sessions were conducted was counterbalanced across pigeons. Between the response-independent and response-dependent blocks, extinction was reestablished for at least five consecutive sessions and until response rates in both components again were below $10 \%$ of baseline for two consecutive sessions. Within each block of four reinstatement sessions, the component (i.e., rich or lean) beginning each session strictly alternated from one session to the next and was counterbalanced across pigeons. Thus, if the rich component was presented during the first session of the block, the lean component was presented first during the next session.

Response rates and reinforcement rates in the rich and lean components were calculated by dividing the number of responses or reinforcers, respectively, by the amount of time spent in the component minus time during reinforcer deliveries. Consistent with the large body of behavioral momentum research on resistance to extinction (for discussions, see Nevin, 1974; Nevin \& Grace, 2000), all statistical analyses of responding during extinction and the reinstatement conditions were conducted on responding as a proportion of baseline response rates. This analysis is also consistent with the suggestion by Baker, Steinwald, and Bouton (1991) that baseline response rates should be normalized before assessing reinstatement of responding. Because the subjects differed in the number of extinction sessions prior to the first reinstatement test, the first four sessions of extinction were used in the analysis of resistance to extinction. In addition, the last day of extinction prior to each reinstatement test was analyzed separately. All statistical tests were considered significant if $p<.05$.

\section{Results and Discussion}

Figure 1 shows mean reinforcement rates on the left $y$-axis and mean responses rates on the right $y$-axis during the last six sessions of baseline training. As was expected, reinforcement rates were higher in the rich component

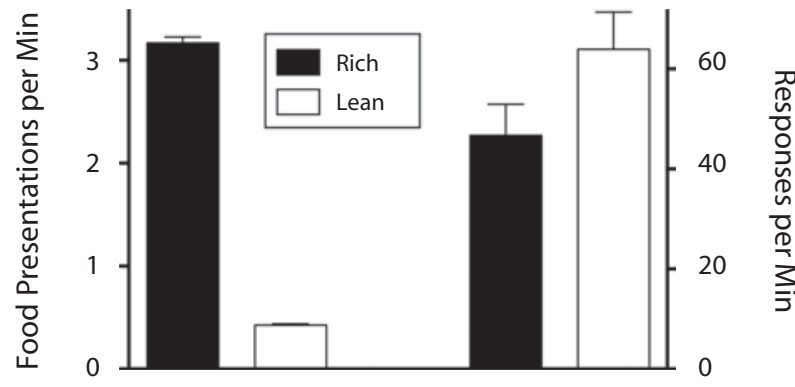

Figure 1. Mean $( \pm S E M)$ food presentations per minute (left $y$-axis) and responses per minute (right $y$-axis) from the last six sessions of baseline for the rich and lean components in Experiment 1.

than in the lean component $[t(9)=49.2]$, and baseline response rates were lower in the rich component than in the lean component $[t(9)=4.557]$. The higher reinforcement rate in the rich component is consistent with an improved stimulus-reinforcer relation in that component. The lower response rates in the rich component are consistent with a degradation of the response-reinforcer relation resulting from the added response-independent reinforcers (see Nevin et al., 1990).

Figure 2 shows responding during extinction and the reinstatement conditions as proportions of baseline response rates in the rich and lean components. Responding in the extinction condition was analyzed with a two-way repeated measures ANOVA (component $\times$ session). Responding decreased significantly across sessions of extinction $[F(4,36)=34.98]$, and resistance to extinction was greater in the rich component than in the lean component, as evidenced by a main effect of component $[F(1,9)=$ $24.01]$ and a significant component $\times$ session interaction $[F(4,36)=8.60]$. Responding did not differ significantly in the rich and lean components on the last day of ex-

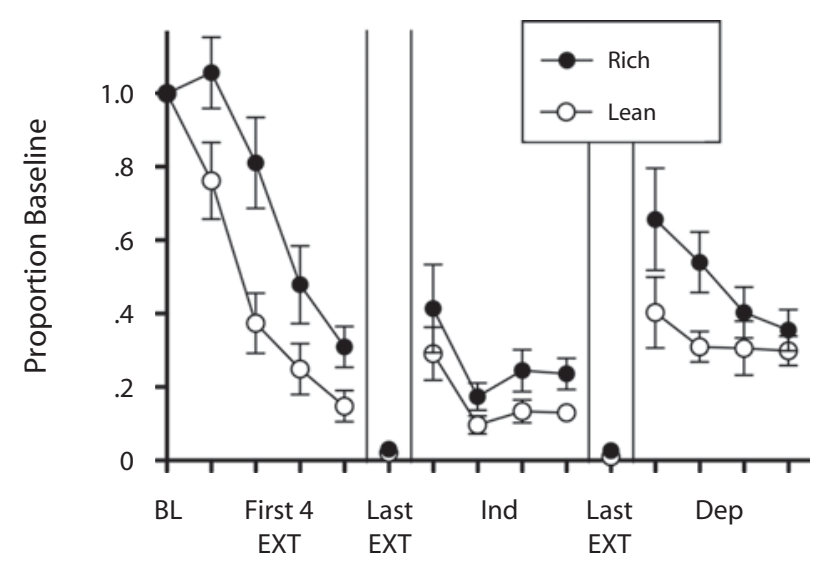

Condition

Figure 2. Mean ( $\pm S E M)$ proportions of baseline (BL) response rates from the first four sessions of extinction (EXT), the last sessions of EXT, and the four sessions of response-independent (Ind) and response-dependent (Dep) food presentations during the reinstatement conditions in Experiment 1. 
tinction prior to the response-independent $[t(9)=1.06]$ or response-dependent $[t(9)=1.46]$ reinstatement tests. But, as compared with the last day of extinction, mean responding in the response-independent condition increased in both components [rich, $t(9)=4.65$; lean, $t(9)=5.88$ ], as did responding in the response-dependent condition [rich, $t(9)=6.95$; lean, $t(9)=6.81$ ] .

Responding during the reinstatement conditions was further analyzed with a three-way repeated measures ANOVA (component $\times$ reinstatement type $\times$ session). A significant three-way interaction between component, reinstatement type, and session was obtained $[F(3,27)=$ 3.25]. Main effects revealed that reinstatement was greater in the rich component than in the lean component $[F(1,9)=9.92]$, reinstatement was greater with response-dependent than with response-independent reinforcement deliveries $[F(1,9)=11.89]$, and responding decreased across reinstatement sessions $[F(3,27)=$ 4.96]. None of the two-way interactions were significant. Further analysis of the three-way interaction revealed that it resulted from a marginally significant component $x$ session interaction for the response-dependent condition $[F(3,27)=2.90, p=.052]$, but not for the responseindependent condition $[F(3,27)=0.41]$. Nonetheless, significant main effects of component were obtained for both the response-independent $[F(1,9)=6.33]$ and response-dependent $[F(1,9)=12.03]$ conditions when considered alone.

These findings replicate the results of previous studies suggesting that the rate of reinforcement in the presence of a stimulus governs relative resistance to extinction (e.g., Nevin et al., 1990). In addition, the present findings suggest that the relative degree of reinstatement following extinction similarly depends on the rate of reinforcement in the presence of a stimulus. This correspondence between resistance to extinction and reinstatement suggests that the relative recovery of discriminated operant behavior could potentially be understood within the larger framework of behavioral momentum theory. The goal of the following two experiments was to assess the generality of the findings from Experiment 1 by assessing other existing methods for examining the relapse of operant behavior following extinction.

\section{EXPERIMENT 2}

This experiment examined the impact of additional response-independent reinforcer presentations on relative resistance to extinction and on the relative increase in behavior produced by a resurgence paradigm. As in Experiment 1 , a baseline condition arranged equal VI schedules for an operant response in the presence of two stimuli within a multiple schedule. In the presence of one stimulus, added response-independent reinforcers were also delivered. Next, the operant response was extinguished, and the added reinforcers were discontinued at the same time that a different operant response started producing reinforcement. The new response was then extinguished, and the original operant response was assessed.

\section{Method}

Subjects and Apparatus. The subjects were 10 unsexed homing pigeons. The pigeons had experience responding on similar schedules of reinforcement (some in the other experiments reported here), and therefore, no preliminary training was needed. The Appendix lists the pigeons serving in each experiment and the order of exposure to the experiments. Pigeons were housed and cared for in the same way, and experimental sessions were conducted in the same operant chambers as in Experiment 1. Both the center and right keys were used in this experiment.

Procedure. The baseline multiple schedule of reinforcement was identical to that arranged in Experiment 1. Thus, pecks to a center key produced reinforcement on separate VI 120 -sec schedules in both the rich and lean components. In addition, responseindependent reinforcers were delivered on a VT 20 -sec schedule in the rich component. Thirty-five sessions were conducted during the baseline condition, after which the extinction condition began. The center keylight remained on, but pecks to the center key were extinguished in both components, and response-independent food presentations were discontinued in the rich component. In addition, the right keylight was turned on in both components and was the same color in both components. During the first session of the extinction condition, the first response on the right key in both components was reinforced. Following the first reinforced response and throughout the remainder of the condition, a VI 30-sec schedule was arranged for responding on the right key in both components. The resurgence condition began once response rates on the center key decreased below $10 \%$ of baseline response rates in both components for a single session. In the resurgence condition, reinforcement for responding on the right key was discontinued in both components for two consecutive five-session blocks. The right key remained lit during the resurgence condition. The component presented first during the initial session in the resurgence condition was counterbalanced across pigeons. After the first session, the component beginning each session strictly alternated for the remainder of the condition. Analyses were the same as those in Experiment 1, with the exception that the first five sessions of extinction were used so as to be consistent with the two blocks of five sessions of resurgence.

\section{Results and Discussion}

Figure 3 shows mean reinforcement rates on the left $y$-axis and mean responses rates on the right $y$-axis during the last six sessions of baseline training. As was expected, reinforcement rates were higher in the rich component than in the lean component $[t(9)=75.11]$, and baseline response rates were lower in the rich component than in the lean component $[t(9)=6.54]$. As in Experiment 1, the higher reinforcement rates and lower response rates

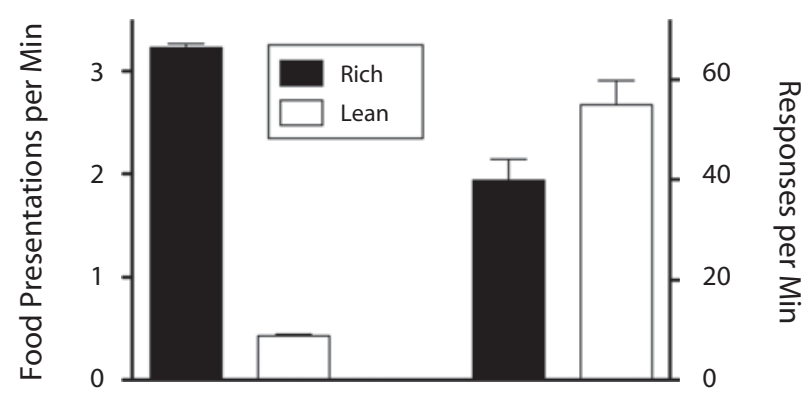

Figure 3. Mean $( \pm S E M)$ food presentations per minute (left $y$-axis) and mean responses per minute (right $y$-axis) from the last six sessions of baseline for the rich and lean components in Experiment 2. 


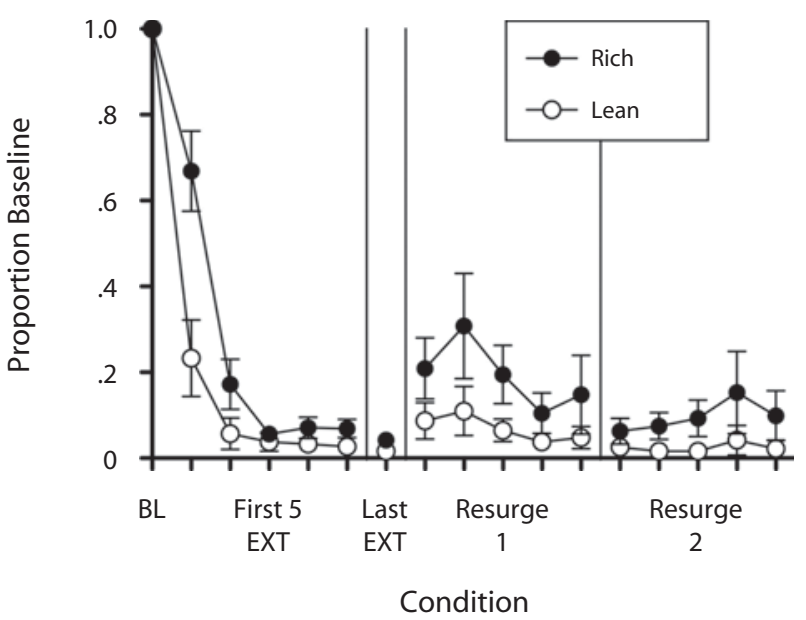

Figure 4. Mean ( $\pm S E M)$ proportions of baseline (BL) response rates from the first five sessions of the extinction (EXT) condition, the last session of EXT, and the first block (Resurge 1) and second block (Resurge 2) of five sessions during the resurgence condition in Experiment 2.

in the rich component are consistent with an improved stimulus-reinforcer relation and a degraded responsereinforcer relation.

Figure 4 shows responding during extinction and the resurgence conditions as proportions of baseline response rates in the rich and lean components. Responding in the extinction condition was analyzed with a two-way repeated measures ANOVA (component $\times$ session). Responding decreased significantly across sessions of extinction $[F(5,45)=103.94]$, and resistance to extinction was greater in the rich component than in the lean component, as evidenced by a main effect of component $[F(1,9)=$ $20.45]$ and a significant component $\times$ session interaction $[F(5,45)=22.51]$. Although responding in the rich and lean components differed significantly $[t(9)=3.5]$ on the last day of extinction prior to the resurgence test, the difference in the components was very small (i.e., difference in proportion of baseline $<.03$ ). As compared with the last day of extinction, mean responding in the resurgence condition increased significantly in the rich component $[t(9)=$ $2.60]$, but the increase only approached statistical significance in the lean component $[t(9)=2.06, p=.07]$.

Responding during the resurgence condition was analyzed with a three-way repeated measures ANOVA (component $X$ resurgence block $X$ session). Resurgence was greater in the rich component than in the lean component, as indicated by a significant main effect for component $[F(1,9)=8.57]$. Resurgence did not differ significantly across five-session blocks $[F(1,9)=2.71]$ or as a function of session $[F(4,36)=0.44]$. In addition, none of the interactions were significant.

As was true in Experiment 1, the finding that the rate of reinforcement in the presence of a stimulus governs relative resistance to extinction is consistent with the results of a large body of research on behavioral momentum (see Nevin \& Grace, 2000). In addition, as was true of reinstatement in Experiment 1, the present findings suggest that relative resurgence following extinction similarly depends on the rate of reinforcement in the presence of a stimulus.

\section{EXPERIMENT 3}

The final experiment examined the effects of added response-independent reinforcers on relative resistance to extinction and ABA renewal of responding after extinction. As in Experiments 1 and 2, a baseline condition arranged equal VI schedules for an operant response in the presence of two stimuli within a multiple schedule. In the presence of one stimulus, added response-independent reinforcers were also delivered. Next, the overall stimulus context of the chamber was changed from a steady houselight during the baseline (i.e., Context A) to a flashing houselight (i.e., Context B) during extinction of the operant response in the presence of both multiple-schedule stimuli. Finally, the steady houselight from the baseline condition was reintroduced (i.e., Context A), and the renewal of responding for the multiple-schedule stimuli was examined under continued extinction.

\section{Method}

Subjects and Apparatus. The subjects were 10 unsexed homing pigeons. The pigeons had experience responding on similar schedules of reinforcement, and therefore, no preliminary training was needed. Some of the pigeons had served in Experiment 1, Experiment 2, or both. The Appendix lists the pigeons serving in each experiment and the order of exposure to the experiments. The pigeons were housed and cared for in the same way, and experimental sessions were conducted in the same operant chambers as in Experiments 1 and 2 .

Procedure. The baseline multiple schedule of reinforcement was identical to that arranged in Experiments 1 and 2. Thus, pecks to a center key produced reinforcement on separate VI 120-sec schedules in both the rich and lean components. In addition, response-independent reinforcers were delivered on a VT 20 -sec schedule in the rich component. Eighty sessions were conducted in the baseline condition, during which the houselight remained on throughout all components and ICIs. Next, the extinction condition began, in which responding was extinguished in both components and response-independent food presentations were discontinued in the rich component. Throughout the entire session (i.e., both components and ICIs) for every session of the extinction condition, the houselight flashed on and off every $0.1 \mathrm{sec}$. The renewal condition began once response rates on the center key decreased below $10 \%$ of baseline response rates in both components for a single session. In the renewal condition, reinforcement continued to be unavailable; however, the steady houselight from the initial baseline condition was reintroduced for four consecutive sessions under continued extinction. Components presented first during the first session of the renewal condition for each pigeon were counterbalanced across pigeons. After the first session, the component beginning each session strictly alternated for the remainder of the condition. Analyses were the same as those in Experiment 1.

\section{Results and Discussion}

Figure 5 shows mean reinforcement rates on the left $y$-axis and mean responses rates on the right $y$-axis during the last six sessions of baseline training. As was expected, reinforcement rates were higher in the rich component than in the lean component $[t(9)=99.05]$, and baseline response rates were lower in the rich component than in the lean component $[t(9)=2.86]$. As in Experiments 1 and 2, the higher reinforcement rates but lower 


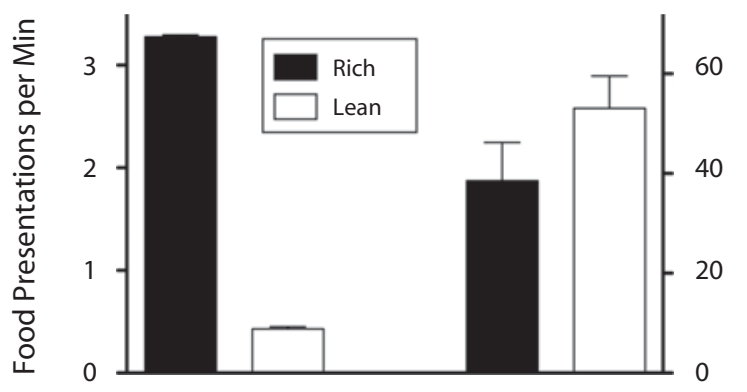

Figure 5. Mean $( \pm S E M)$ food presentations per minute (left $y$-axis) and mean responses per minute (right $y$-axis) from the last six sessions of baseline for the rich and lean components in Experiment 3.

response rates in the rich component are consistent with an improved stimulus-reinforcer relation and a degraded response-reinforcer relation.

Figure 6 shows responding during extinction and the renewal condition as a proportion of baseline response rates in the rich and lean components. Responding in the extinction condition was analyzed with a two-way repeated measures ANOVA (component $\times$ session). Responding decreased significantly across sessions of extinction $[F(4,36)=42.26]$, and resistance to extinction was greater in the rich component than in the lean component, as evidenced by a main effect of component $[F(1,9)=$ $21.82]$ and a significant component $\times$ session interaction $[F(4,36)=6.53]$. Responding did not differ significantly in the rich and lean components on the last day of extinction prior to the renewal test $[t(9)=0.9]$. As compared with the last day of extinction, mean responding in the renewal condition increased for both the rich $[t(9)=5.77]$ and lean $[t(9)=4.75]$ components.

Responding during the renewal condition was analyzed with a two-way repeated measures ANOVA (component $X$ session). Although responding decreased across sessions of the renewal condition $[F(3,27)=10.54]$, renewal was

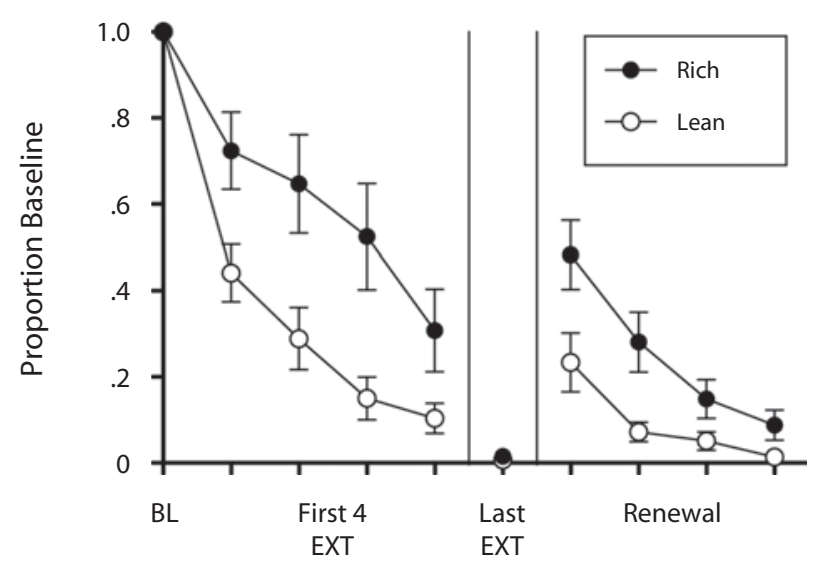

Condition

Figure 6. Mean $( \pm S E M)$ proportions of baseline $(\mathrm{BL})$ response rates from the first four sessions of extinction (EXT), the last session of EXT, and the renewal condition in Experiment 3. greater in the rich component than in the lean component, as indicated by a significant main effect for component $[F(1,9)=18.66]$ and a significant component $\times$ session interaction $[F(3,27)=8.23]$.

These findings are consistent with the results of the previous two experiments, suggesting that stimulus-reinforcer relations govern relative resistance to change and the reappearance of operant responding following extinction. In addition, this experiment provides an additional demonstration of ABA renewal of operant behavior.

\section{GENERAL DISCUSSION}

The present experiments replicate numerous prior experiments showing that adding response-independent reinforcers in the presence of a stimulus increases relative resistance to extinction in the presence of that stimulus, even if those added reinforcers decrease baseline response rates (e.g., Nevin et al., 1990). This finding is consistent with the suggestion from behavioral momentum theory that relative resistance to extinction of operant responding is governed by the Pavlovian relation between a discriminative-stimulus and reinforcers occurring during that stimulus. Most important, the present experiments suggest that relative relapse of operant responding in reinstatement, resurgence, and ABA renewal paradigms is also greater in the presence of a stimulus previously associated with a higher rate of reinforcement, even if some of those reinforcers are delivered independently of the operant response. Thus, the present findings suggest that, as with resistance to extinction, relapse of operant responding appears to depend on the baseline stimulus-reinforcer relation.

A recent series of experiments by da Silva, Maxwell, and Lattal (2008) may at first appear to be inconsistent with the suggestion that relapse of operant behavior depends on the baseline stimulus-reinforcer relation. Da Silva et al. examined resurgence of responding on concurrent schedules of reinforcement with pigeons and, across three experiments, found that relative resurgence of extinguished responding was similar for concurrent responses regardless of differences in baseline reinforcement rates for the two responses. However, behavioral momentum theory suggests that the overall stimulus-reinforcer relation in which an operant response occurs determines resistance to disruption, not the rate of reinforcement produced by a particular response. Because concurrent operant responses share the same stimulus-reinforcer relation, the theory predicts that there should be no difference in resistance to change for the two responses (see Nevin \& Grace, 2000). The fact that da Silva et al. found that relative resurgence of concurrent operants occurring in the presence of the same stimulus was similar despite different response rates and/or reinforcement rates is consistent with the role of the stimulus-reinforcer relation in relapse suggested by the present experiments.

To more formally understand how behavioral momentum theory might be useful for considering how reinforcement rates in the presence of a stimulus affect relapse following extinction, we will examine the account of extinction provided by the theory and suggest how it might be extended to relapse-like phenomena. The augmented 
model of extinction provided by behavioral momentum theory (e.g., Nevin \& Grace, 2000) suggests that the disruptive effects of extinction on responding in the presence of a stimulus may be characterized as

$$
\log \left(\frac{B_{t}}{B_{\mathrm{o}}}\right)=\frac{-t(c+d r)}{r^{b}},
$$

where $B_{t}$ is response rate at time $t$ in extinction, $B_{0}$ is asymptotic response rate in the preextinction baseline, $c$ is the disruptive effect of terminating the contingency between responding and reinforcement, $d$ scales the disruptive impact of the removal of reinforcers (i.e., generalization decrement), $r$ is the rate of reinforcement in the presence of the stimulus in the baseline condition, and $b$ is parameter representing sensitivity to reinforcement rate. Thus, responding in the presence of stimuli associated with higher rates of reinforcement (i.e., $r$ ) is relatively more resistant to the combined disruptive impact of factors represented in the numerator of the equation. The inclusion of parameters for the disruptive effects of suspension of the contingency (i.e., $c$ ) and for the generalization decrement produced by eliminating reinforcer deliveries (i.e., $d$ ) has allowed the model to describe a number of extinction-related phenomena, including the partial reinforcement extinction effect.

In a general sense, the account of extinction provided by Equation 2 is compatible with a common approach to understanding relapse phenomena. Specifically, the reappearance of extinguished behavior characteristic of reinstatement, resurgence, and renewal has been interpreted to suggest that the learning associated with both operant and Pavlovian conditioning preparations survives extinction (e.g., Bouton, 2004). Similarly, Equation 2 also suggests that extinction of operant behavior does not result from unlearning but, rather, is a disruption of ongoing behavior. Furthermore, Bouton and colleagues have noted the critical role played by the context in which extinction takes place. They have argued that postextinction relapse phenomena reflect the effects of a change in the context in which extinction took place. In addition, relapse appears to depend on the associative value of the relevant context (Baker et al., 1991; Bouton \& King, 1983). As was noted above, behavioral momentum theory posits a critical role for the value of the contextual Pavlovian stimulus-reinforcer relation in resistance to extinction - an approach that appears to be consistent with the fact that relapse-like phenomena after extinction depend on the associative value of the context.

One approach to extending Equation 2 to the increase in responding produced by the relapse phenomena studied here is to characterize relapse as resulting from a decrease in the size of the disruptor represented in the numerator with the onset of the relapse operation. Thus,

$$
\log \left(\frac{B_{t}}{B_{\mathrm{o}}}\right)=\frac{-t(m c+m d r)}{r^{b}},
$$

where all the terms are as in Equation 2 and the added parameter $m$ is a scaling factor used to reduce the disruptive effects of contingency suspension (i.e., $c$ ) and generalization decrement associated with eliminating reinforcer deliveries (i.e., $d r$ ). During extinction, $m=1$, and thus
Equation 3 is the same as Equation 2 and predicts greater resistance to extinction in the presence of a stimulus previously associated with a higher rate of reinforcement. With the onset of a relapse operation, $m$ takes a value less than 1 , thus reducing the magnitude of the disruptor in the numerator and producing an increase in responding. As is true with Equation 2 during extinction, Equation 3 predicts that response rates as a proportion of baseline during relapse should be greater in the presence of a stimulus previously associated with a higher rate of reinforcement. The use of the scaling parameter $m$ is similar to an approach previously taken by Nevin and Grace (2005) to capture an abrupt increase in disruption when extinction was implicitly signaled by the absence of a reinforcer that usually occurred at a particular time. To understand the implications of using the parameter $m$ to decrease the disruptive impact of extinction with the onset of a relapse-producing event, recall that such phenomena are thought to reflect the fact that the effects of extinction are relatively specific to the stimulus conditions present during extinction. Thus, $m$ might be viewed as a means to scale a reduction in the disruptive impact of extinction associated with a change in stimulus conditions present during extinction.

Figure 7 shows a simulation of relative resistance to extinction and relative relapse based on Equation 3. For ease of exposition, the antilog of the predictions of Equation 3 are presented and correspond to responding as a proportion of baseline. Parameter values for the simulation were $c=$ 2.0, $d=0.001, b=0.5$, and $m=1$ in extinction versus $m=$ 0.25 in relapse. Predictions for rich and lean components were generated by using the reinforcement rates arranged in the present experiments (e.g., rich $=210$ reinforcers $/ \mathrm{h}$, lean $=30$ reinforcers $/ h$ ). As was expected, the model predicts greater resistance to extinction in the rich component than in the lean component. In addition, the model predicts not only an increase in responding with the onset of the relapse operation, but also a greater increase in responding relative to baseline in the rich component than in the lean component. Thus, Equation 3 appears to produce predictions consistent with the effects of baseline reinforcement rate on both relative resistance to extinction and the relative increase in responding produced by relapse.

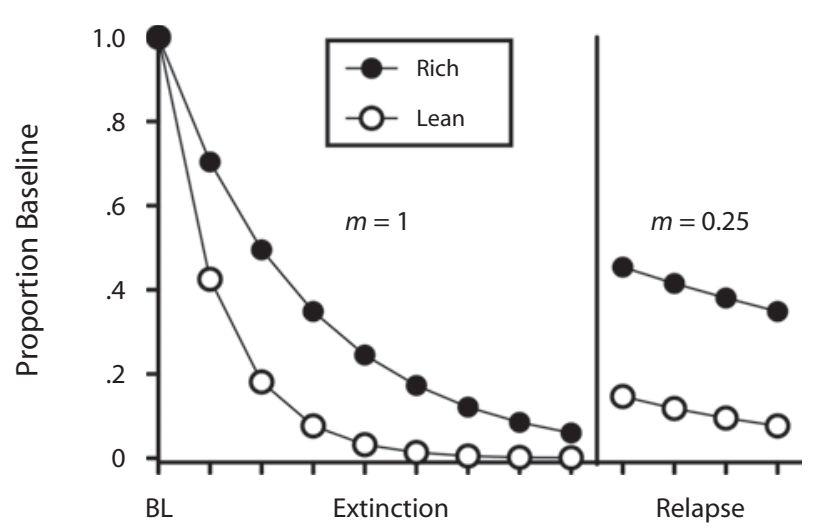

Figure 7. Simulation of relative resistance to extinction and relapse generated by Equation 3. Details are provided in the text. 
Although the details of how Equation 3 should be extended specifically to relapse in reinstatement, resurgence, and renewal procedures might differ, the model in principle should be applicable to the effects of differences in baseline reinforcement rates on relapse in general. Regardless, the basic account would be the same: Relapse reflects a reduction in the disruptive effects of extinction resulting from an implicit or explicit change in stimulus conditions associated with the extinction context. Thus, behavioral momentum theory as formalized in Equations 3 may be useful as an account of the effects of baseline reinforcer rates on the relapse of operant responding.

\section{AUTHOR NOTE}

Portions of this work were funded by National Institute on Alcohol Abuse and Alcoholism Grant R01AA016786 to T.A.S. The authors thank Amy Odum and Tony Nevin for their comments and Scott Barrett, Adam Kynaston, and Abby Brinkerhoff for their assistance in conducting these experiments. Correspondence concerning this article should be addressed to T. A. Shahan, Department of Psychology, Utah State University, 2810 Old Main Hill, Logan, UT 84322 (e-mail: tim.shahan@usu.edu).

\section{REFERENCES}

Ahearn, W. H., Clark, K. M., Gardenier, N. C., Chung, B. I., \& DubE, W. V. (2003). Persistence of stereotyped behavior: Examining the effects of external reinforcers. Journal of Applied Behavior Analysis, 36, 439-448.

Baker, A. G., Steinwald, H., \& Bouton, M. E. (1991). Contextual conditioning and reinstatement of extinguished instrumental conditioning. Quarterly Journal of Experimental Psychology, 43B, 199-218.

Bouton, M. E. (2004). Context and behavioral processes in extinction. Learning \& Memory, 11, 485-494.

Bouton, M. E., \& King, D. A. (1983). Contextual control of the extinction of conditioned fear: Tests for the associative value of the context. Journal of Experimental Psychology: Animal Behavior Processes, 9, 248-265.

CoHen, S. L. (1996). Behavioral momentum of typing behavior in college students. Journal of Behavior Analysis \& Therapy, 1, 36-51.

Da Silva, S. P., Maxwell, M. E., \& Lattal, K. A. (2008). Concurrent resurgence and behavioral history. Journal of the Experimental Analysis of Behavior, 90, 313-331.

EPSTEIN, R. (1996). Cognition, creativity, and behavior: Selected essays. Westport, CT: Praeger.

Fleshler, M., \& Hoffman, H. S. (1962). A progression for generating variable-interval schedules. Journal of the Experimental Analysis of Behavior, 5, 529-530.

Grimes, J. A., \& Shull, R. L. (2001). Response-independent milk delivery enhances persistence of pellet-reinforced leverpressing by rats. Journal of the Experimental Analysis of Behavior, 76, 179-194.

HARPER, D. N. (1999). Drug-induced changes in responding are dependent on baseline stimulus-reinforcer contingencies. Psychobiology, 27, 95-104.

HERRNSTEIN, R. J. (1970). On the law of effect. Journal of the Experimental Analysis of Behavior, 13, 243-266.

IGAKI, T., \& SAKAGAMI, T. (2004). Resistance to change in goldfish. Behavioural Processes, 66, 139-152.

Leitenberg, H., Rawson, R. A., \& BAth, K. (1970). Reinforcement of competing behavior during extinction. Science, 169, 301-303.

Leitenberg, H., Rawson, R. A., \& Mulick, J. A. (1975). Extinction and reinforcement of alternative behavior. Journal of Comparative \& Physiological Psychology, 88, 640-652.

Mace, F. C., Lalli, J. S., Shea, M. C., Lalli, E. P., West, B. J., Rob-
ERTS, M., \& NeVIN, J. A. (1990). The momentum of human behavior in a natural setting. Journal of the Experimental Analysis of Behavior, 54, 163-172.

Nakajima, S., Tanaka, S., Urushinara, K., \& Imada, H. (2000). Renewal of extinguished lever- press responses upon return to the training context. Learning \& Motivation, 31, 416-431.

Nevin, J. A. (1974). Response strength in multiple schedules. Journal of the Experimental Analysis of Behavior, 21, 389-408.

Nevin, J. A. (1992). An integrative model for the study of behavioral momentum. Journal of the Experimental Analysis of Behavior, 57, 301-316.

Nevin, J. A., \& GraCE, R. C. (2000). Behavioral momentum and the law of effect. Behavioral \& Brain Sciences, 23, 73-130.

Nevin, J. A., \& Grace, R. C. (2005). Resistance to extinction in the steady state and in transition. Journal of Experimental Psychology: Animal Behavior Processes, 31, 199-212.

Nevin, J. A., Tota, M. E., Torouato, R. D., \& Shull, R. L. (1990). Alternative reinforcement increases resistance to change: Pavlovian or operant contingencies? Journal of the Experimental Analysis of Behavior, 53, 359-379.

Podlesnik, C. A., Jimenez-Gomez, C., \& Shahan, T. A. (2006). Resurgence of alcohol seeking produced by discontinuing non-drug reinforcement as an animal model of drug relapse. Behavioural Pharmacology, 17, 369-374.

REID, R. L. (1958). The role of the reinforcer as a stimulus. British Journal of Psychology, 49, 202-209.

SeE, R. E. (2002). Neural substrates of conditioned-cue relapse to drug-seeking behavior. Pharmacology, Biochemistry, \& Behavior, 71, 517-529.

Shaham, Y., Shalev, U., Lu, L., De Wit, H., \& Stewart, J. (2003). The reinstatement model of drug relapse: History, methodology, and major findings. Psychopharmacology, 168, 3-20.

Shahan, T. A., \& Burke, K. A. (2004). Ethanol-maintained responding of rats is more resistant to change in a context with added non-drug reinforcement. Behavioral Pharmacology, 15, 279-285.

WELKER, R. L., \& MCAULEY, K. (1978). Reductions in resistance to extinction and spontaneous recovery as a function of changes in transportational and contextual stimuli. Animal Learning \& Behavior, 6, 451-457.

\section{APPENDIX}

Stimuli Used for Each Experiment and the Order of Exposure to the Experiments for Individual Pigeons

\begin{tabular}{rllll}
\hline & \multicolumn{3}{c}{ Stimulus } & \\
\cline { 2 - 4 } Pigeon & Lean & Rich & Right Key* & Experiment \\
\hline 56 & yellow & turquoise & red & $3,1,2$ \\
68 & turquoise & yellow & white & 2 \\
121 & white & yellow & red & $3,2,1$ \\
218 & white & yellow & & 3 \\
219 & blue & white & & 3 \\
234 & turquoise & blue & white & 2,1 \\
237 & green & white & turquoise & 2,1 \\
284 & white & green & red & 2,1 \\
586 & yellow & red & white & 2,3 \\
658 & yellow & turquoise & & 3,1 \\
927 & white & yellow & red & $3,2,1$ \\
938 & red & yellow & white & $2,1,3$ \\
1803 & blue & turquoise & yellow & 2,1 \\
1877 & turquoise & yellow & & 3,1 \\
9178 & yellow & red & & 3 \\
\hline
\end{tabular}

${ }^{*}$ Experiment 2 only.

(Manuscript received April 2, 2009; revision accepted for publication June 17, 2009.) 